\title{
Bloqueo ecoguiado esp en el manejo del dolor postoperatorio en cirugía bariátrica
}

\author{
Cáceres J. ${ }^{1}$, Alonso R. ${ }^{1,2}$, Crippa NA. ${ }^{1}$ \\ 1 Hospital San Roque, La Plata, Argentina. \\ 2 Instituto Médico Platense, La Plata, Argentina.
}

Introducción: El ESP block (bloqueo del erector espinal) es un bloqueo interfascial que consiste en la inyección de solución anestésica entre la fascia de los músculos erectores de la columna y las apófisis transversas vertebrales. La evidencia respalda que la difusión paravertebral y peridural de la solución inyectada brinda bloqueo somático, visceral y simpático.

Objetivos y Métodos: Se comparó retrospectivamente la percepción de dolor en pacientes a los que se les realizó esta técnica anestésica en el preoperatorio de cirugía bariátrica, en relación a pacientes que no la recibieron. Para eso, se revisaron las historias clínicas del Servicio de Anestesia. Se consideraron dos grupos: aquellos que habían recibido el bloqueo ESP guiado por ecografía, y aquellos que no lo recibieron. Criterios de inclusión: pacientes adultos, que recibieron cirugía bariátrica, que hayan aceptado recibir el bloqueo y no presenten signos de infección en la zona de trabajo, en condiciones neurológicas aptas para comprender preguntas y comunicarse correctamente.

El bloqueo se realizó con el paciente sentado, bajo anestesia general, con colaboración de asistentes. ESP block bilateral, a la altura de T6. La solución de elección fue bupivacaína $0,25 \% 20 \mathrm{ml}$. por lado. Aquellos que no recibieron el ESP block, solamente recibieron anestesia general.

Se utilizó la escala numérica verbal para evaluar el dolor (ENV)[1] a las 24 horas de realizado el bloqueo.

Resultados: Se incluyeron cinco pacientes con ESP block y 8 sin ESP. La distribución por sexo fue tres mujeres y dos hombres para los dos grupos, siendo el rango etario entre 31 y 56 años.

Se vio que aquellos que recibieron el ESP presentaron significativamente menos dolor que aquellos que no lo recibieron a las $24 \mathrm{~h}$ (postoperatorio) $(\mathrm{p}=0,0476$ : IC95\% $=3,97-6,02)$ (Tabla 1$)$.

Conclusión: El ESP block demostró ser útil en el manejo del dolor post cirugía bariátrica, sin necesidad de utilizar analgésicos opioides en el postoperatorio y evitando los efectos secundarios de los mismos en este grupo de pacientes.

Referencias: 1. Downie WW, Leatham PA, Rhind VM, Wright V, Branco JA, Anderson JA. Studies with pain rating scales. Ann Rheum Dis. 1978 Aug;37(4):378-81.

https://doi.org/10.25237/congresoclasa2019.51 